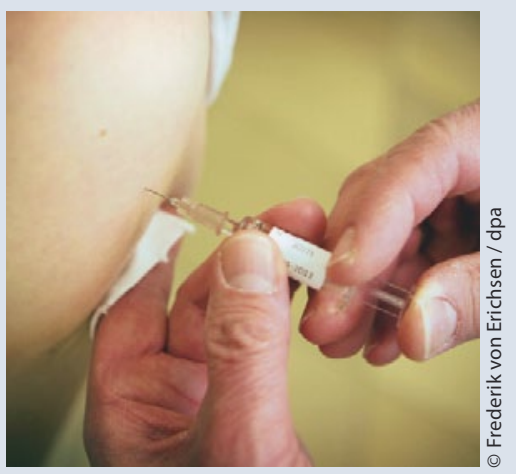

\section{Narkolepsie als Impfschaden}

Nicht nur Vireninfektionen, sondern auch Impfungen könnten eine Narkolepsie mit Kataplexie triggern.

Es gibt immer mehr Hinweise auf einen Zusammenhang zwischen viralen Infektionen und der Entwicklung einer Kataplexie, berichtete Professor Christian Baumann, Zürich. Inzwischen verdichtet sich aber auch der Verdacht, dass die Schweinegrippe-Impfung mit Pandemrix ${ }^{\circledast}$ bei der Entstehung von Narkolepsie insbesondere bei Kindern und Jugendlichen eine Rolle spielt. Aktuell belegt eine britische retrospektive Untersuchung einen möglichen kausalen Zusammenhang [Miller E et al. BMJ 2013; 346: f794]. In Finnland, wo sehr viele Kinder und Jugendliche betroffen sind, wurde die Narkolepsie laut Baumann bereits als Impfschaden anerkannt.

Meldungen von Verdachtsfällen sind in Deutschland von Arzt oder Betroffenen/ Verbrauchern beim Paul Ehrlich-Institut (PEI) möglich [www.pei.de, Menü Vigilanz/Pharmakovigilanz]. Nach einer Mitteilung vom 13. März 2013 erhielt das Paul Ehrlich-Institut zwischen Oktober 2010 und 25. Februar 2013 aus Deutschland insgesamt 37 Meldungen von Narkolepsieverdachtsfällen nach der Pandemrix-Impfung, davon bei 22 Kindern und Jugendlichen im Alter von acht bis 17 Jahren und 15 Erwachsenen. Die "Ständige Impfkommission" (STIKO) empfiehlt bereits seit der vorletzten Saison die Verwendung des nicht verdächtigten trivalenten saisonalen Grippeimpfstoffs. Das PEl selbst führt derzeit eine Fall-Kontroll-Studie zu Risikofaktoren der Narkolepsie durch [Oberle D et al. Somnologie 2013, in Druck].

Friederike Klein

Baumann C: Narkolepsie. Vortrag im Rahmen des Symposiums "Schlaf- und Bewegungsstörungen". Deutscher Parkinson-Kongress 2013, Würzburg, 15.3.2013
Double. Dafür könnte eine veränderte Methylierung der entsprechenden Genabschnitte verantwortlich sein, aber auch ein Kupfermangel durch eine kupferstimulierte Aggregation von a-Synuclein und Neuromelanin, wie von ihrer Arbeitsgruppe mittels Röntgen-Mikrofluoreszenzmikroskopie gezeigt werden konnte. Ein therapeutisches Ziel könnte daher sein, den Kup- fergehalt im SN zu normalisieren. Da der Ablauf der Neuromelaninsynthese in vivo noch nicht geklärt ist, kommt er als therapeutisches Ziel derzeit noch nicht infrage.

Friederike Klein

Präsidentensymposium "Neurobiologie von Neuromelanin und dessen mögliche Pathofunktion bei der Parkinsonerkrankung". Deutscher Parkinson-Kongress 2013, Würzburg, 14.3.2013

\title{
Neuroprotektion muss früher stattfinden
}

\section{Studienteilnehmer sollten bereits aufgrund subklinischer neurologischer Veränderungen ausgewählt werden.}

$\mathrm{B}$ isherige Versuche, in Studien eine neuroprotektive Wirkung von Medikamenten eindeutig nachzuweisen, schlugen fehl. Die Ansätze kamen einfach zu spät, ist Professor Günter Höglinger, München, überzeugt. Vor der frühen klinisch-symptomatischen Phase, in der in Studien behandelt wird, liegen lange Jahre subklinischer neurologischer Veränderungen. Zukünftig müssen sich daher Studien stärker auf Risikopopulationen, etwa mit Hyposmie, REMSchlaf-Verhaltensstörung (RBD) oder Obstipation konzentrieren. Die Identifi- zierung der Konversionsraten in solchen Risikogruppen ermöglicht dann Interventionsstudien mit dem primären Endpunkt der relativen Risikoreduktion der Entwicklung eines klinischen Morbus Parkinson. Als neuroprotektive Therapieziele kommen dann eine ganze Reihe von älteren und neueren Wirkprinzipien infrage (Tabelle 1). $\quad$ Friederike Klein

Höglinger G: Krankheitsmodifizierende Therapie. Symposium „Medikamentöse Innovationen der Parkinson-Therapie". Deutscher ParkinsonKongress 2013. Würzburg, 14.3.2013.

\section{Potenzielle Möglichkeiten einer Neuroprotektion bei PD}

\section{Ansatzpunkte für} Neuroprotektion

Oxidativer Stress

Mitochondriale

Dysfunktion

Excitotoxizität

Inflammation

Apoptose

Neuronale Dysfunktion

Protein-Degradation

Anti-Spreading

Anti-Aggregativa

\section{Wirkprinzip}

Antioxidanzien

Bioenergetische Substanzen

NMDA-Antagonisten

Antiinflammatorisch wirkende Substanzen

Antiapoptotisch wirkende Substanzen

Trophische Faktoren

Antikörper
Beispiele

Vitamin E, Vitamin C, Fe-Chelatoren Coenzym Q10

MK801 (Dizocilpine)

Cox-2-Inhibitoren, PPRy

Dopaminagonisten, Caspase-Inhibitoren, AKT GDNF, Nurturin, Neuregulin Proteosomal Enhancer, Rapamycin EGCG (Polyphenol aus Grünem Tee), ANLE 